\title{
Estudo da regulação transcricional do COL18A1 e análise funcional do domínio Frizzled.
}

Aluna: Erika Kague

Orientadora: Profa. Dra. Maria Rita Passos-Bueno 


\section{Abstract}

The completion of multiple vertebrate genome sequences has presented an important challenge to understand and predict function from primary DNA sequence, particularly for non-coding sequence. It is commonly hypothesized that evolutionary conservation predicts functional DNA sequences, including those involved in regulating gene transcription, although sequence conservation has proven to be an imperfect predictor of enhancer function. Type XVIII collagen is a component of most basement membranes; mutations in the COL18A1 gene lead to Knobloch Syndrome, an autosomal recessive disease characterized by vitreoretinal and macular degeneration and occipital encephalocele. COL18A1 has 43 exons that transcribe three isoforms from two different promoters. The three isoforms display complex patterns of tissue-specific expression, including in kidney, lung, brain, and retina. Expression levels of COL18A1 are thought to be clinically important in vasculogenesis, and in predisposition to hepatocarcinoma and diabetes type 2 . Therefore, identification of the regulatory regions will provide insight into normal and pathogenic regulation of COL18A1 expression. Furthermore, endostatin and FRZC18 are cleaved fragments from collagen XVIII that are involved in Wnt signaling, however in vivo role of FRZC18 has not been investigated yet in any model organism. Thus, a deeper investigation of FRZC18 role in Wnt signaling is indubitable necessary, as well as a comprehension of COL18A1 regulation by Wnt signaling. We have employed an efficient system of transgenesis in the zebrafish to functionally evaluate potential enhancer elements regulating COL18A1 transcription. We identified four enhancer elements that control transcription consistent with zebrafish endogenous coll8al, in tissues including retina, kidney, blood vessels, gut, cartilage 
and liver. Although the algorithms we used did not detect non-coding conservation from human to teleosts at the COL18A1 locus, the human sequences functioned appropriately in zebrafish transgenics. Additional post hoc computational analysis revealed detectable sequence similarities between human and zebrafish in or near two of the four enhancer regions. We tested one of these zebrafish regions and confirmed orthologous enhancer activity. We functionally tested FRZC18 with its mRNA overexpression in zebrafish embryos. This experiment resulted in embryos with phenotype remaining slb and ppt, mutants of non-canonical wnt components. This result points FRZC18 as an antagonist of non-canonincal Wnt signaling possibly by interaction with Wnt11 and Wnt5. We dissected COL18A1 promoter 1 and it showed characteristics of a housekeeping gene and similarities with promoter 2 and we also showed possible TCF/LEF binding to COL18A1 promoters. Wnt signaling responded to downregulate promoter activity of COL18A1 and also decrease its expression by overexpression of $\beta$-catenin. This work broadly elucidated COL18A1 cis regulatory elements and better characterized its role in Wnt signaling as an antagonist of noncanonical and also as a target of canonial signaling. 


\section{Resumo}

A conclusão do seqüênciamento do genoma de múltiplos vertebrados trouxe um importante desafio para entender e predizer função, particularmente para seqüências não-codificantes, a partir de seqüências primarias de DNA. A hipótese de que a conservação evolutiva prediz seqüências funcionais é comumente aceita, inclusive para seqüências envolvidas na regulação da transcrição gênica, mesmo que a conservação de seqüências tenha gerado imperfeitas predições de enhancers (acentuadores) funcionais. O colágeno tipo XVIII é um componente da maioria das membranas basais; mutações no gene COL18A1 levam a síndrome de Knobloch, uma doença autossômica recessiva caracterizada por degeneração vitreoretiniana e macular e encefalocele occipital. O COL18A1 tem 43 exons que transcrevem três isoformas a partir de dois promotores diferentes. As três isoformas apresentam um complexo modelo de expressão tecido-específico, incluindo expressão em rim, pulmão, cérebro e retina. Os níveis de expressão do COL18A1 são considerados clinicamente importantes na vasculogenese, e em predisposição para o hepatocarcinoma e diabetes tipo 2. Dessa forma, a identificação de regiões regulatórias fornecerá indícios sobre a regulação da expressão do COL18A1 em estados normal e patogênico. Além disso, a endostatina e o FRZC18 são fragmentos proteolíticos do colágeno XVIII envolvidos na sinalização Wnt. No entanto, o papel in vivo do FRZC18 ainda não foi estudado. Uma profunda investigação do papel deste domínio na via de sinalização Wnt é indubitavelmente necessária, bem como a compreensão da regulação do COL18A1 pela via de Wnt. Empregamos um sistema eficiente de teste em zebrafish para analisar o potencial funcional de elementos enhancers na regulação transcricional do COL18A1. Identificamos quatro elementos enhancers que controlam a transcrição consistente com o coll8al endógeno de zebrafish, em tecidos incluindo retina, rim, 
vasos sanguíneos, intestino, cartilagem e fígado. Apesar dos algoritmos utilizados não tenham detectado conservação em seqüências não-codificantes de humanos à teleósteos no lócus do COL18A1 estudado, as seqüências humanas funcionaram apropriadamente em zebrafish transgênicos. Adicionais análises computacionais post hoc revelaram similaridade entre seqüência humana e de zebrafish dentro ou próximo das quatro regiões enhancers. Testamos funcionalmente o FRZC18 com superexpressão de seu RNAm em embriões de zebrafish. Este experimento resultou em embriões com fenótipos que assemelharam à mutantes da via não-canônica de Wnt (slb e ppt). Este resultado aponta o FRZC18 como um antagonista da via de sinalização não-canônica de Wnt, possivelmente por interação com Wnt11 e Wnt5. Dissecamos o promotor 1 do COL18A1, o qual mostrou características de genes "housekeeping" e similaridades com o promotor 2 do COL18A1. Também mostramos possível ligação de TCF/LEF aos promotores do COL18A1. A via de Wnt levou à redução de atividade dos promotores do COL18A1 e também redução dos níveis de expressão através de superexpressão de $\beta$-catenina. Este trabalho elucidou de forma geral, os elementos regulatórios em cis do COL18A1 e melhor caracterizou o seu papel na via de sinalização Wnt como um antagonista também da via não-canônica e como um alvo da via canônica. 


\section{Introdução}

\subsection{Colágenos}

Os colágenos são elementos estruturais encontrados em tecidos conectivos e intersticiais, virtualmente em todos os órgãos parenquimais, representando os constituintes mais abundantes da matriz extracelular (Prockop e Kivirikko, 1995). Os membros da família dos colágenos são caracterizados por apresentarem um domínio central de colágeno (COL) formado por repetições triplas de Gly-Xaa-Yaa (as posições Xaa e Yaa são frequentemente ocupadas por prolina e hidroxiprolina) relacionadas com a formação da estrutura de triplas hélices; no entanto os colágenos variam em tamanho, função e distribuição tecidual (Gelse, Poschl et al., 2003). Domínios não-colagenosos (NC) podem ser encontrados em regiões terminais de alguns tipos de colágenos, como no colágeno $\mathrm{XV}$, e ausentes em outros tipos, como no colágeno I. Cerca de 26 colágenos, geneticamente distintos, foram descritos até o momento (Myllyharju e Kivirikko, 2001). A família dos colágenos pode ser dividida em dois grupos, fibrilares e não-fibrilares. Os colágenos fibrilares (Ex: tipos I, II, III, $\mathrm{V}$ e XI) garantem flexibilidade e resistência aos tecidos e representam aproximadamente $90 \%$ de todos os colágenos. Os colágenos não - fibrilares (Ex: tipos IV, X, XII, XIV, XV e XVIII) apresentam grande heterogeneidade em estrutura, localização e função (Prockop e Kivirikko, 1995; Gelse, Poschl et al., 2003). 


\subsection{Colágeno tipo VXIII}

O colágeno XVIII pertence ao grupo dos colágenos não - fibrilares, formando junto com o colágeno XV o subgrupo das MULTIPLEXINAS (múltiplos domínios triplas hélices com interrupções) (Oh, Kamagata et al., 1994; Oh, Warman et al., 1994). O cDNA do colágeno XVIII foi clonado em 1993/94, permitindo a elucidação da cadeia $\alpha 1$ de sua estrutura primária (Abe, Muragaki et al., 1993; Oh, Warman et al., 1994; Rehn e Pihlajaniemi, 1994), esta formada por polipeptídeos com 11 domínios não - colagenosos (NC1-NC11) e 10 domínios colagenosos (COL1-COL10) (Oh, Warman et al., 1994; Rehn, Hintikka et al., 1994). O colágeno XVIII é uma heparan-sulfato proteoglicana e possui uma variante polipeptídica caracterizada pela presença de três domínios não-colágenos na porção $\mathrm{N}$-terminal, diferente do colágeno XV, uma condroitil - sulfato proteoglicana (Saarela, Ylikarppa et al., 1998).

O gene COL18A1 foi mapeado no cromossomo 21q22.3, apresenta aproximadamente $105 \mathrm{~kb}$ e inclui 43 exons, sendo que o intron 2 corresponde a quase metade do tamanho total do gene (Oh, Warman et al., 1994). Seu ortólogo em camundongos está localizado no cromossomo 10 e apresenta características similares ao humano (102 kb total e 43 exons) (Rehn, Hintikka et al., 1994). Foram identificadas 3 isoformas do colágeno XVIII e a transcrição para essas variantes ocorre através de dois promotores alternativos, o promotor 1 localizado "upstream" ao exon 1 e codifica a isoforma curta do colágeno XVIII, denominada NC1-303 com 1336 aminoácidos (aa). Esta isoforma é formada pelos exons 1, 2 e $4-43$. O promotor 2 está localizado "upstream" ao exon 3 e codifica a isoforma longa (NC1728 com 1751 aa) e média (NC1-493 com 1516 aa), ambas formadas pelos exons 3 e 4 - 43. O exon 3 apresenta um "splicing" alternativo responsável pela formação de 
dois transcritos que variam em tamanho, os RNAs das isoformas longa e média. Visto que, as isoformas curta e longa apresentam a mesma porção carboxi-terminal no exon 43, a diferença existente entre elas é o peptídeo sinal na porção amino-terminal (Elamaa, Snellman et al., 2003).

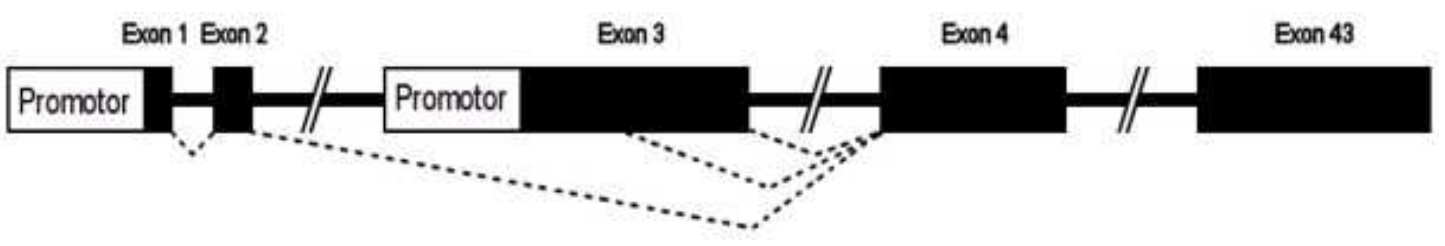

Figura 1. Esquema do gene COL18A1, com dois promotores, 43 exons e 3 isoformas distintas (linhas tracejadas). A isoforma longa é gerada por "splicing" no exon 3 (Figura modificada de (Suzuki, Sertie et al., 2002).

Em geral, o colágeno XVIII é expresso em rim, fígado, pulmão e onipresente em membranas basais (Rehn, Hintikka et al., 1994; Halfter, Dong et al., 1998; Saarela, Rehn et al., 1998; Saarela, Ylikarppa et al., 1998). Porém, a existência de dois promotores alternativos no gene COL18A1 possibilita a expressão diferenciada das variantes do colágeno entre os tecidos. Assim como em camundongos, a variante NC1- 303 de humanos (homóloga a variante NC1- 301 de camundongos) é encontrada predominantemente no rim e em níveis inferiores no coração, placenta e parede endotelial (Saarela, Ylikarppa et al., 1998), além disso, esta isoforma também foi encontrada na retina e cérebro (Sertie, Sossi et al., 2000). A variante NC1-493 é expressa principalmente no fígado e a variante $\mathrm{NC1}-728$ no pulmão, fígado e em menores concentrações no coração, cérebro e em músculo esquelético fetal (Muragaki, Timmons et al., 1995; Saarela, Rehn et al., 1998; Saarela, Ylikarppa et al., 1998). A expressão do colágeno XVIII já foi investigada em outras espécies mais distantes evolutivamente, como galinha e zebrafish, e um padrão de expressão similar foi verificado. Em zebrafish o collsal foi detectado em placa neural anterior e células adaxiais (estágio de 5 somitos), cérebro anterior e mediano, notocorda, 
espinha cordal, epiderme, ductos pronéfricos, mioseptum, vesícula ótica, campo ocular e primórdios de nadadeira peitoral (estágio de 24hpf a 48hpf) (Haftek, MorvanDubois et al., 2003). Em galinhas o coll8al foi detectado em retina, cápsula das lentes, epiderme, rim, pulmão, gânglio e nervos do sistema nervoso periférico, espinha cordal e vasos sanguineos (Halfter, Dong et al., 1998).

A função do colágeno XVIII ainda não é bem definida em todos os tecidos em que o mesmo é expresso, no entanto, trabalhos têm demonstrado a relação deste colágeno com algumas doenças, evidenciando sua importância para o bom funcionamento de alguns tecidos e órgãos. Em 2000 Sertié e colaboradores, verificaram que uma mutação no intron 1 do gene COL18A1 causa a Síndrome de Knobloch (SK), e neste caso há comprometimento somente da isoforma curta. Este trabalho demonstrou pela primeira vez que o gene do COL18A1 é o responsável pela etiologia desta síndrome e confirmou que esta doença faz parte do grupo das colagenopatias (Sertie, Quimby et al., 1996; Sertie, Sossi et al., 2000).

A Síndrome de Knobloch é uma desordem autossômica recessiva definida pela ocorrência de alta miopia, degeneração vitreoretiniana com descolamento da retina, anormalidades maculares e encefalocele occipital. Existe uma variabilidade clínica entre os afetados, porém, todos os pacientes apresentam anormalidades oculares severas. A encefalocele também é um dos aspectos clínicos mais observados (Czeizel, Goblyos et al., 1992; Passos-Bueno, Marie et al., 1994). Em 2002 foram identificadas novas mutações patogênicas relacionadas ao fenótipo da SK, sugerindo que as outras isoformas do colágeno XVIII também poderiam desempenhar um papel crítico na organização e manutenção do olho humano (Suzuki, Sertie et al., 2002). 
As anormalidades oculares encontradas em pacientes com a SK sugerem um papel importante do COL18A1 principalmente em estruturas oculares. Além disso, defeitos em migração neuronal também foram relatados em pacientes com KS. Caenorhabditis elegans com falta de colágeno XVIII apresentam defeitos em migração celular e direcionamento axonal (Ackley, Crew et al., 2001), indicando o papel funcional do colágeno XVIII também na migração neuronal. Mas molecularmente não se conhece o mecanismo que o colágeno XVIII possa estar envolvido na migração neuronal.

Camundongos nocautes para o colágeno XVIII apresentam atraso na regressão dos vasos hialóides após o nascimento e outros defeitos oculares, como atrofia das células epiteliais ciliares, descolamento do epitélio pigmentar da retina e redução da pressão intra-ocular dependente do avanço da idade (Fukai, Eklund et al., 2002; Marneros e Olsen, 2003). Os camundongos nocautes (Coll8a1 \%) também têm ampla membrana basal dos túbulos renais, alteração na matriz mesangial dos gromérulos e elevado nível de creatinina no plasma, e ainda pode desempenhar um papel na suscetibilidade a danos renais associados a diabetes (Utriainen, Sormunen et al., 2004). Dessa forma, é incontestável o importante papel do colágeno XVIII em diferentes tecidos, porém ainda pouco se conhece sobre regulação de sua expressão tecido-específico e mecanismos moleculares envolvidos.

\subsubsection{Fragmentos clivados do Colágeno VXIII}

A endostatina e o Frizzled (FRZC18) são fragmentos proteolíticos do colágeno XVIII, representando domínios de alta conservação com outras espécies. A 
endostatina apresenta 20- KDa e é derivada dos últimos 184 resíduos da porção Cterminal do colágeno XVIII, região com cerca de $99 \%$ de homologia entre humanos e camundongos (Saarela, Ylikarppa et al., 1998). A endostatina tem capacidade de inibir a proliferação de células endoteliais, angiogênese, crescimento metastático e também inibir o crescimento em tumores primários (O'reilly, Boehm et al., 1997). Pacientes com Síndrome de Down, os quais têm menor incidência de tumores sólidos, mostraram níveis plasmáticos elevados de endostatina, indicativo de ação como agente protetor para o desenvolvimento de tumores sólidos (Zorick, Mustacchi et al., 2001). O polimorfismo D104N localizado no domínio da endostatina foi recentemente associado ao câncer de mama esporádico (Lourenco, Cardoso-Filho et al., 2006). A endostatina tem sido investigada quanto ao seu poder anti-angiogênico em diversos tumores e também em estudos terapêuticos (Wu, Ding et al., 2008; Hu, Kou et al., 2009; Retsky, Hrushesky et al., 2009).

Uma segunda região proteolítica do colágeno XVIII de alta conservação entre humanos, camundongos e Xenopus é o domínio Frizzled (FRZC18), derivado da porção N-terminal da variante longa (Elamaa, Snellman et al., 2003). O FRZC18 apresenta 120 aminoácidos contendo uma seqüência de 10 cisteínas invariavelmente espaçadas, "cysteine rich domain" (CRD). A região FRZC18 é homóloga a parte ligante dos receptores envolvidos na via de sinalização Wnt e proteínas solúveis relacionadas ao Frizzled (sFRPs). As sFRPs são proteínas antagonistas que ligam-se diretamente aos Wnts ou a seus receptores (Kawano e Kypta, 2003). Recentemente, o FRZC18 foi descrito como inibidor da via de sinalização Wnt/ß-catenina, com potencial de diminuir crescimento de células tumorais através do aumento de morte celular em linhagens celulares de câncer humano com ativação de ß-catenina (Quelard, Lavergne et al., 2008). A endostatina quando injetada em embriões de 
xenopus leva a inibição da via de wnt/ ß-catenina através de indução da degradação citoplasmática (Hanai, Gloy et al., 2002). Dessa forma, os dois fragmentos proteolíticos do colágeno XVIII demonstram capacidade de interferência na via de sinalização Wnt, porém estudos adicionais são necessários para melhor detalhamento e função do colágeno XVIII na via de sinalização Wnt. O COL18A1 ainda não foi estudado como alvo de ß-catenina, um ponto importante para responder questões pertinentes ao envolvimento do colágeno XVIII e seus fragmentos proteolíticos nesta via de sinalização.

\subsection{Sinalização Wnt}

Os Wnts compreendem uma família conservada de moléculas glicoprotéicas de sinalização envolvidas em desenvolvimento e processos biológicos, incluindo proliferação, diferenciação e polaridade celular (Logan e Nusse, 2004). A sinalização Wnt é agrupada em duas classes: canônica e não-canônica (Baksh e Tuan, 2007).

O mecanismo principal da sinalização canônica é dependente de estabilização de ß-catenina no citoplasma, ativando genes alvos dos fatores de transcrição TCF/LEF. Na ausência de sinalização Wnt, ocorre ubiquitinação de ß-catenina e sua degradação pelo proteosomo. O complexo citoplasmático de degradação que se liga a ß-catenina é consistindo em pelo menos Axina, proteína APC e GSK-3. A ativação da via canônica inicia-se com ligação de Wnt ao respectivo receptor Frizzled e a correceptores LRP5-6 ("LDL receptor related protein"), transduzindo sinal para Dishevelled (Dsh) e para Axina, levando a inibição da fosforilação de ß-catenina e inibição de sua degradação. Os níveis elevados de ß-catenina no citoplasma leva ao 
seu acúmulo no núcleo e formação de complexo ativador com os fatores de transcrição TCF/LEF e consequentemente, indução transcricional de genes alvos. Mutações que promovem a ativação constitutiva da via canônica podem levar ao câncer (Logan e Nusse, 2004). O melhor exemplo conhecido é a Polipose Familiar Adenomatosa (FAP), uma doença autossômica dominante em que os pacientes apresentam pólipos no cólon e ativação exacerbada de ß-catenina (Ponti, Losi et al., 2008). Mutações que levam a perda de função em Axina foram descritas em hepatocarcinomas (Taniguchi, Roberts et al., 2002). Ativação da via Wnt está envolvida com numa grande variedade de tipos tumorais (Logan e Nusse, 2004; Doucas, Garcea et al., 2005). Além disso, o aumento e diminuição de ß-catenina tem papel importante durante osteogêneses e adipogênese, incitando e inibindo respectivamente (Bowers e Lane, 2008; Errera, Canani et al., 2008; Pederson, Ruan et al., 2008).

A via não-canônica envolve outras proteínas não relacionadas com $\beta$-catenina, e ativa vias de sinalização como a "planar cell polarity" (PCP) que guia movimentos durante a gastrulação e via de $\mathrm{Wnt} / \mathrm{Ca}^{2+}$ (Topczewski, Sepich et al., 2001; Katoh, 2007). Os Wnts ligam-se diretamente aos receptores Frizzled levando a alterações em localização de proteína efetoras da via, como JNK, Dvl e ROCKII ou alterações em concentrações de cálcio intracelular (Habas, Kato et al., 2001; Kishida, Yamamoto et al., 2004; Endo, Wolf et al., 2005; Rosso, Sussman et al., 2005). Durante a embriogênese a via não-canônica participa da determinação de polarização dorsoventral dos membros. Apesar dos efeitos desta via serem menos conhecidos, estudos indicam a participação desta via em migração neuroral. O papel do FRZC18 ainda não foi estudado na via não - canônica de Wnt, e a implicação do FRZC18 nesta via 
poderia ajudar a entender a função do COL18A1 na migração neuronal, já que pacientes com SK, com ausência da isoforma longa, apresentam defeitos em migração neuronal. A investigação da participação do FRZC18 na via não-canônica poderia ajudar a esclarecer o papel do colágeno XVIII em processos importantes como a migração neuroral.

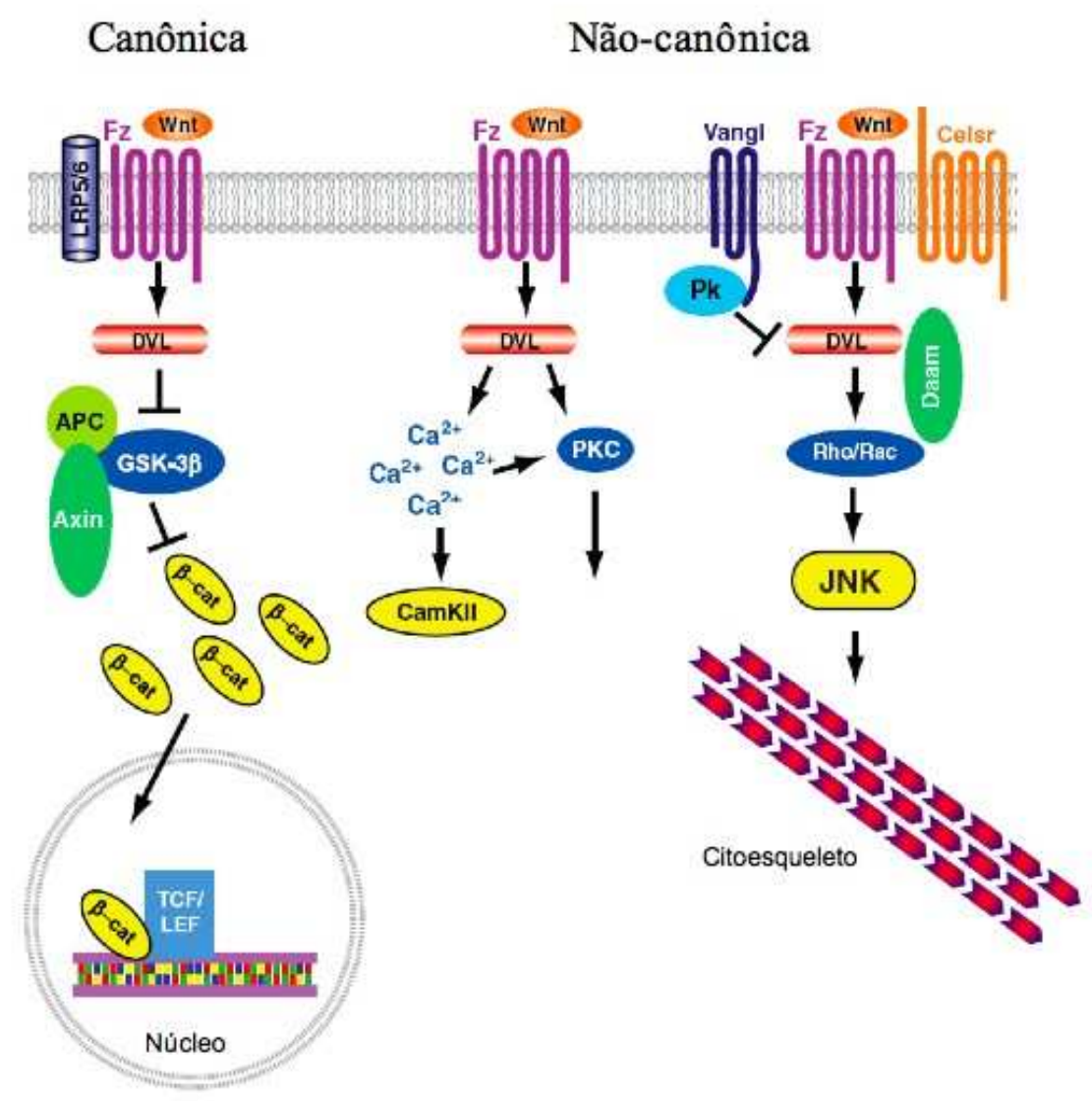

Figura 2. Via de sinalização Wnt canônica e não-canônica. (Figura modificada de (Montcouquiol, Crenshaw et al., 2006).

\subsection{Regulação Gênica Transcricional}

Os Eucariotos empregam diversos mecanismos para controle de expressão gênica, incluindo condensação de cromatina, metilação de DNA, iniciação transcricional, "splicing" alternativo de RNA, estabilidade de mRNA, controles 
traducionais, diversas formas de modificações pós-traducionais, tráfego intracelular e degradação de proteína. Dentre essas categorias gerais, a regulação transcricional é um dos primeiros mecanismos controlador da atividade espaço-temporal dos genes e em eucariotos a regulação é realizada através de interação de múltiplos fatores de transcrição (FT) (elementos em trans) com elementos cis, incluindo "enhancers", “silencers", "insulators" e promotores. O promotor está localizado "upstream” ao sítio de início da transcrição e serve como ponto de reconhecimento por fatores de transcrição básicos de recrutamento da RNA polimerase II (Ex. TFIIA, TFIIB e TFIIF). A região controladora de genes típicos de eucariotos apresenta regiões promotoras proximais (“core promoter" - promotor basal) e distais (“enhancers" elementos regulatórios positivos ou acentuadores). Tipicamente, o termo promotor refere ao "core promoter" e as seqüências adjacentes (“enhancers proximais"). O “core promoter” é a região mínima necessária para transcrição gênica basal reconhecidas pela RNA Polimerase II, localiza-se imediatamente à 5' do sitio iniciador de transcrição e contém as seqüências canônicas suficientes pelo para reconhecimento da maquinaria transcricional e iniciação da transcrição. Os “enhancers" proximais apresentam sítios de transcrição tecido-específicos bem como os elementos distais de regulação ("enhancers").

Devido a importância de regiões promotoras e "enhancers" mutações e polimorfismos nessas regiões podem acometer a funcionalidade gênica e taxa de expressão gênica. Mutações em sítios de ligação para fatores de transcrição nas regiões promotoras podem levar a redução transcricional de genes, como observado por Niimi e colaboradores, em estudo relacionando mutação no promotor do gene UGRP1 com menor transcrição do mesmo e maior risco à asma (Niimi et al., 2002). Lettice e colaboradores, demonstraram que a polidactilia preaxial resulta de mutações 
de ponto em região reguladora "enhancer" do gene $S h h$, concluindo que mutações de ponto nestas regiões são capazes de causar anormalidades congênitas (Lettice et al., 2003). O promotor 2 do COL18A1 foi caracterizado previamente e mostrou características de um gene "housekeeping", com ausência de TATA box e rico em GC. Sp1, Sp3 e YY1 foram fatores de transcrição identificados para regulação deste promotor e um polimorfismo na região está relacionado com diferença de expressão em hepatócitos (Armelin-Correa, Lin et al., 2005). Porém, o promotor 1 do COL18A1 ainda não foi estudado. E nada se conhece sobre elementos regulatórios deste gene. Diferenças na taxa de expressão do COL18A1 estão relacionadas com patologias como o hepatocarcinoma e diabetes tipo 2. Desta forma, o estudo da regulação transcricional deste gene, bem como a identificação de elementos regulatorios em cis, ajudariam a entender variações de expressão gênica em diferentes patologias e também seria uma tentativa a inferir sobre causa da variabilidade fenotîpica encontrada em pacientes portadores da Síndrome de Knobloch.

Apesar da importância de seqüências regulatórias, uma porcentagem ínfima de seqüências não-codificantes funcionais foram identificadas até o momento. O projeto ENCODE ("ENCyclopedia Of DNA Elements") representa a preocupação da comunidade cientifica em melhor entender a localização de elementos regulatórios em tentativa de melhor conhecer o funcionamento da regulação de expressão gênica (The ENCODE (ENCyclopedia Of DNA Elements) Project, 2004). A identificação de elementos regulatórios não-codificantes representa um grande desafio. Uma comparação entre o genoma humano e de camundongo mostrou que $40 \%$ do genoma pode ser alinhado. E estima-se que 5\% do genoma de mamíferos está sob seleção e predito para serem funcionais. Surpreendentemente, desses 5\% apenas 1,5\% correspondem exons e o restante é representado por seqüências não-codificantes. $\mathrm{Ou}$ 
seja, seqüências não-codificantes e conservadas correspondem em mais que o dobro das seqüências codificadoras de proteína no genoma (Pennacchio, 2003). Uma hipótese atual de que seqüências funcionais são conservadas entre as espécies abriu uma janela de estudo e busca de regiões não - codificantes funcionais através de comparação entre seqüências de diferentes espécies, principalmente aquelas que compartilham uma fisiologia ou biologia comum, como humanos-camundongos. A comparação de sequencias com outros organismos ( por exemplo, galinhas e peixes, como fugu e zebrafish) é interessante para ajudar a delimitar sequencias funcionais quando a região restudada tem grande conservação entre humanos e camundongos (Nobrega e Pennacchio, 2004). Como exemplo, podemos destacar a identificação de “enhancer” específico de tecido cardíaco através de comparação entre seqüências de humano, camundongo e galinha e delimitação de uma região de alta similaridade de seqüência e após testes funcionais em camundongos a comprovação que a região regula o gene Nkx2-5 (Lien, Mcanally et al., 2002). Outros estudos foram realizados envolvendo regiões conservadas evolutivamente. Regiões pobres em genes e maiores que $500 \mathrm{~kb}$ chamadas de desertos gênicos podem conter elementos regulatórios com habilidade de modular a expressão gênica a distância. O gene $D A C H$ é separado de outros genes por desertos gênicos e para a identificação de regiões conservadas evolutivamente nesses desertos gênicos, Nóbrega e colaboradores alinharam a região com vertebrados, incluindo sapo, zebrafish e puferfish e encontraram 32 regiões conservadas. Em testes funcionais com camundongos reduziram o número para 7 regiões que dirigem a expressão de $\beta$-galactosidase em diferentes tecidos (Nobrega, Ovcharenko et al., 2003). Em 2005 o grupo caracterizou "in vivo" o "enhancer" Dc2 do gene $D A C H$, este “enhancer" tem identidade de $82 \%$ humano-fugu e $424 \mathrm{pb}$, sendo que apresenta um bloco de importância funcional de 144 pb (Poulin, Nobrega et al., 
2005). Bejerano e colaboradores alinharam seqüências de humanos, camundongos e ratos, e identficaram 481 segmentos maiores que 200 pb e com $100 \%$ de identidade entre as seqüências, estas regiões foram chamadas de regiões ultraconservadas (Bejerano, Pheasant et al., 2004). Dessa forma, comparação entre seqüências de espécies diferentes têm sido uma ferramenta importante para a identificação de regiões não-codificantes funcionais no genoma e poderia ser aplicada para identificação de elementos regulatórios em cis do COL18A1.

Para estudo funcional das regiões não-codificantes e conservadas são utilizados modelos "in vitro" e modelos "in vivo". O camundongo tem sido bastante empregado para estes estudos (Nobrega, Ovcharenko et al., 2003; Poulin, Nobrega et al., 2005). Galinha também é outro modelo animal que pode se utilizado (Uchikawa, Ishida et al., 2003). Porém, atualmente o modelo animal que se destaca é o zebrafish (Pashos, Kague et al., 2008).

\subsection{Zebrafish como modelo animal}

Dentre os modelos animais que se destacam atualmente para estudos funcionais, o Zebrafish (Danio rerio) é um dos mais utilizados. Inúmeras razões incentivam o uso deste modelo como, o seu tamanho pequeno, fácil manutenção dos aquários, rápida reprodução, transparência dos embriões e o alto número de embriões gerados por um único casal (Shin, Priest et al., 2005; Fisher, Grice, Vinton, Bessling, Urasaki et al., 2006). O grande número de mutantes produzidos em curto período de tempo através de mutágenos químicos e a disponibilidade do sequênciamento do genoma dessa espécie, bem como ricos bancos de dados disponíveis para consulta de seqüência e expressão gênica (Ex. ZFIN- "The Zebrafish Model Organism 
Database"), também fazem desde modelo um alvo ideal para estudos funcionais de domínios protéicos, genes e regiões genômicas não-codificantes, além de estudos envolvendo genética e embriologia. A disponibilidade de programas de bioinformática capazes de promover o alinhamento entre seqüências de diversas espécies e inferências de similaridade gênica nos proporcionam uma útil ferramenta de estudo de genes humanos em outras espécies. Alinhamento entre mamíferos e espécies mais distantes evolutivamente, como peixes, podem representar potencial funcional, como já observado em diversos estudos (Shin, Priest et al., 2005; Mcgaughey, Vinton et al., 2008). Recentemente verificou-se que a identificação de "enhancers" pode ser realizada utilizando o modelo de peixe sem que exista similaridade de seqüência entre humano e teleósteos que os programas em geral são incapazes de detectar (Fisher, Grice, Vinton, Bessling e Mccallion, 2006; Fisher, Grice, Vinton, Bessling, Urasaki et al., 2006). Isto torna o modelo de zebrafish ainda mais interessante para identificação de elementos regulatórios funcionais.

Além de estudos funcionais envolvendo seqüências não-codificantes, o zebrafish pode ser utilizado para entendimento de vias de sinalização. A disponibilidade de mutantes para proteínas envolvidas nas vias de sinalização é uma grande vantagem deste modelo. Zebrafish mutantes Slb/Wnt11 e ppt/Wnt5a, envolvidos com sinalização não-canônica, foram descritos com participação de movimentos de convergência e extensão e PCP durante a gastrulação em desenvolvimento embrionário de zebrafish (Heisenberg, Tada et al., 2000; Kilian, Mansukoski et al., 2003). Mutantes homozigotos para Slb apresentam regiões anteriores afetadas no embrião, mostrando transientemente encurtamento e alargamento do eixo corporal no final da gastrulação seguido de fusão dos olhos em estágios mais avançados de desenvolvimento. Em contraste, os mutantes homozigotos 
ppt exibem um encurtamento do eixo corporal em estágios tardios da gastrulação enquanto que a posição dos olhos não é afetada. Slb e ppt exibem funções parcialmente sobrepostas em regulação de movimentos de extensão e convergência (CE) em domínios laterais da gástrula, como em duplo nocaute ppt, o fenótipo homozigoto de $s l b$ é mais grave. Embriões de zebrafish injetados com $\beta$-catenina ou proteínas envolvidas na via canônica levam à duplicação de eixo nos embriões, respostas fenotípicas distintas resultantes de ativação da via canônica e não-canônica da sinalização Wnt (Kelly, Erezyilmaz et al., 1995).

\subsection{Objetivos}

Considenrando a regulação transcricional do colágeno XVIII, apenas o promotor 2 foi estudado até o momento. Um estudo mais amplo de regulação transcricional do COL18A1 não somente ajudaria a entender processos relacionados com o COL18A1, mas também enriqueceria o conhecimento geral sobre regulação transcricional, sobretudo a identificação de seqüências funcionais não-codificantes, um assunto relativamente novo e pouco conhecido. Com exceção do domínio da endostatina, a função que o colágeno exerce através do FRZC18 ainda continua pouco explorada e pouco conhecida. E apesar do envolvimento do colágeno XVIII na via de sinalização Wnt ter sido sugerido, o papel do colágeno XVIII na via ainda é obscuro.

Dessa forma, neste trabalho procuramos identificar elementos regulatórios em cis do COL18A1 utilizando-se o modelo de zebrafish; dissecar o promotor 1 do COL18A1; explorar se o COL18A1 é um gene regulado pela $ß$-catenina e também, a investigação funcional "in vivo” do domínio Frizzled do colágeno XVIII (FRZC18) na via de sinalização

Wnt. 
Capítulo VI- Discussão

Capítulo VI 


\section{Discussão}

O colágeno XVIII é um importante componente da maioria das membranas basais do corpo e tem um complexo modelo de expressão, com participação em diversos tecidos e órgãos durante o desenvolvimento embrionário e na fase adulta (Halfter, Dong et al., 1998; Elamaa, Peterson et al., 2002; Kawashima, Watanabe et al., 2003). O COL18A1 apresenta dois promotores que dirigem a transcrição de três isoformas distintas com picos de expressões em diferentes tecidos. Mutações que levam à perda de função do gene COL18A1 causam a Síndrome de Knobloch, cuja as características fenotípicas marcantes envolvem problemas oculares, como alta miopia e descolamento de retina e também a presença de encefalocele occipital (Sertie, Sossi et al., 2000; Suzuki, Sertie et al., 2002). A endostatina como produto proteolítico do colágeno XVIII é um potente inibidor de angiogenese e tem sido investigada para utilização em terapias gênicas (O'reilly, Boehm et al., 1997; Hong, Lee et al., 2004). O colágeno XVIII também foi recentemente vinculado ao processo de adipogenese, com aumento de expressão do mesmo durante a diferenciação adipogênica, e predisposição em patogêneses como diabetes tipo 2 e hepatocarcinoma (Musso, Rehn et al., 2001; Errera, Canani et al., 2008). A participação deste colágeno como um antagonista na via de Wnt através de fragmentos proteolíticos, endostatina e Frizzled, é uma questão recente e pouco conhecida funcionalmente e molecularmente (Hanai, Gloy et al., 2002; Quelard, Lavergne et al., 2008).

Visto o importante papel do COL18A1 e inúmeras questões pertinentes a sua complexa função e distribuição tecidual, uma melhor elucidação da regulação deste gene e sua participação funcional na via de Wnt são extremamente relevantes. Desta forma, neste trabalho procuramos entender a participação in vivo do FRZC18 e a regulação transcricional do $C O L 18 A 1$, estudando não apenas as regiões basais de 
regulação transcricional, mas investigando também seqüências reguladoras de caráter genômico, os enhancers distais.

O promotor 1 COL18A1, até então ainda não caracterizado, apresenta uma região mínima capaz de promover a transcrição basal de luciferase entre -103 e $275 \mathrm{pb}$ do sitio de início da transcrição. Esta região, ou promotor basal, tem similaridades com o promotor 2 do COL18A1 (Armelin-Correa, Lin et al., 2005). Ambas seqüências são ricas em sítios de ligação para o fator de transcrição Sp1 e são ausentes de TATA ou CAAT boxes, característica de genes "housekeeping” (CAP IV). A semelhança entre os promotores basais poderia ajudar a entender a sobreposição de expressão basal referente a cada isoforma. Além do promotor basal, identificamos três seqüências que aumentam a atividade do promotor, PE1-3. A região PE3 mostrou-se funcional em testes in vivo, confirmando a região como um enhancer proximal. Apesar de PE3 induzir relativamente uma maior atividade em células HepG2 e indicando regulação tecido-específico, expressão em fígado não foi observada in vivo. Análises de alinhamento e conservação entre a seqüência promotora ortóloga de camundongos seguidas de predição para fatores de transcrição, delimitamos alguns fatores de transcrição que poderiam desempenhar papel regulador nos PEs, entre eles TCF/LEF, PPRA-gamma, AP-2 e NF3 que estão envolvidos em via de Wnt, adipogenese, formação de crista neural e alta expressão em fígado, respectivamente. Mostramos que os fatores de transcrição TCF/LEF potencialmente podem se ligar à região promotora do $C O L 18 A 1$, e que aumento de $\beta$-catenina resulta em redução de atividade dos promotores do COL18A1 e redução de sua expressão. Dessa forma, essa relação inversa entre colágeno XVIII e ß-catenina nos ajuda a melhor entender e especular um pouco sobre a regulação molecular em processos como a adipogenese e também o câncer. Durante a adipogenese é observado uma redução na expressão de 
Wnt1 (Ross, Hemati et al., 2000), um ativador de ß-catenina. E concomitantemente, observa-se um aumento de expressão de COL18A1 (CAP IV). Este fato corrobora a regulação negativa do COL18A1 pela ß-catenina. Além disso, diferenças em níveis de expressão de COL18A1 está diretamente ligada a níveis de endostatina e FRZC18, ambos antatonistas de ß-catenina. Em cânceres onde a via de Wnt está comprometida com aumento exacerbado de ß-catenina, poderíamos imaginar uma redução de COL18A1 e consequentemente, redução de endostatina e aumento de crescimento tumoral. Dessa forma, o estabelecimento de ligação entre o colágeno XVIII como um gene alvo regulado pela via de Wnt ajuda a entender processos biológicos importantes e complexos em que relativamente pouco se conhece molecularmente.

O modelo complexo de expressão do COL18A1 estende-se desde nemátodos, até humanos. O colágeno XVIII desempenha papel em migração neuronal em nemátodos e em migração de axônios motores em zebrafish também foi descrito (Ackley, Crew et al., 2001; Schneider e Granato, 2006). Sua expressão em galinhas muito se assemelha à encontrada em zebrafish e em camundongos (Halfter, Dong et al., 1998; Haftek, Morvan-Dubois et al., 2003). Neste trablho, descrevemos expressão de COL18A1 em tecidos embrionários de zebrafish que ainda não haviam sido ralatados, como fígado, cartilagem e vasos sanguíneos (CAP III). Em embriões de camundongos o COL18A1 apresenta o mesmo padrão de expressão observado em galinhas e em zebrafish, o que indica e corrobora o complexo modelo de expressão do COL18A1 e conservação de regulação da expressão desse gene ao longo da evolução. Nós identificamos quatro distintos elementos regulatórios em cis do COL18A1 (CAP III) que somando-se dirigem expressão em tecidos de zebrafish representando quase completamente a expressão endógeno deste gene, incluindo ducto pronéfrico, fígado, retina, vasos sanguíneos, cartilagem, intestino, notocorda, cérebros anterior, mediano 
e posterior, arcos faringeais e vesícula ótica. A expressão dirigida por cada elemento sugere regulação isoforma-específica, já que CNSv -97.8/ CNSp -96.9 leva expressão em ducto pronéfrico enquanto que $\mathrm{CNSv}+64.8$ em fígado, onde as isoformas curtas e media/longa são altamente expressas, respectivamente. $\mathrm{CNSp}+47.8$ dirige expressão em vasos sangüíneos e pode ser uma seqüência alvo para estudos relacionados com aumento de expressão de endostatina. Os resultados também indicam que os enhancers responsáveis por regulação tecido-específico estão dispostos distintamente do promotor do gene.

As construções testadas funcionalmente como enhancers foram selecionadas de acordo com conservação entre mamíferos e não apresentaram conservação com espécies distantes evolutivamente, como fugo, stickleback e zebrafish. Porém, interessantemente, as seqüências funcionaram em zebrafish. Submetemos as regiões já identificadas funcionalmente em analises post hoc utilizando uma ferramenta com maior sensibilidade porém, incapaz de calcular significância ou a conservação da vizinhança e que geralmente fornece maior número de falso-positivos. Identificamos conservação com zebrafish nas seqüências das regiões CNS -96.9 e CNSp +47.8. Testamos funcionalmente a seqüência de zebrafish ortóloga a CNSp +47.8, e obtivemos expressão mosaica em vasos sanguíneos, resultados que se sobrepõe à respectiva construção humana. Este resultado indica que as regiões funcionais sem conservação detectada com primeira analise, ainda apresentam seqüências detectadas por fatores de transcrição comuns e conservados entre zebrafish e mamíferos e podem funcionar em zebrafish, complementando também trabalho anterior realizado por Fisher e colaboradores (Fisher, Grice, Vinton, Bessling e Mccallion, 2006; Fisher, Grice, Vinton, Bessling, Urasaki et al., 2006). 
Além de analizarmos a regulação transcricional do COL18A1 identificando enhancers e caracterizando o promotor 1 do gene, também testamos in vivo o papel do domínio FRZC18. Através de superexpressão deste domínio em embriões de zebrafish, relacionamos o mesmo com a via não -canônica de Wnt. A via canônica, dependende de $\beta$-catenina, é a mais conhecida e estudada e pouco se entende sobre a via não-canônica, porém alterações nas divergentes vias são claramente diferenciadas fenotipicamente em embriões de zebrafish. Defeitos na via canônica de Wnt em embriões de zebrafish, promovem duplicação aterior de eixo (Kelly, Erezyilmaz et al., 1995). Enquanto que alteração na via não-canônica relaciona-se com encurtamento de eixo posterior e redução de distancia interocular nos embriões (Heisenberg, Tada et al., 2000; Kilian, Mansukoski et al., 2003; Ulrich, Concha et al., 2003). Os resultados indicaram participação de FRZC18 na via não-canônica de Wnt (CAP V), sendo que os embriões apresentaram características fenotípicas semalhantes a mutantes wnt11 e wnt5, slb e ppt, respectivamente. Isto implica participação do FRZC18 em movimento de gastrulação durante a embriogênese. Observamos defeitos marcantes de gastrulação nos embriões, quando injetados com FRZC18, incluindo enlargamento de notocorda e somitos. Interessantemente, não observamos alteração na via canônica de Wnt que envolve ß-catenina. Recentemente, o mesmo domínio foi relacionado funcionalmente como antagonista de $\beta$-catenina em linhagens celulares de hepatocarcinoma (Quelard, Lavergne et al., 2008). Dessa forma, além da via de ßcatenina o colágeno XVIII também participa da via não- canônica possivelmente com interação com as proteínas Wnt11 e Wnt5. Participam da via não-canônica proteínas como ROCKII e JNK e a reorganização do citoesqueleto é o resultado efetivo da via. Como participante de matriz extracelular em membranas basais o colágeno XVIII estruturalmente poderia levar à alterações no citoesqueleto através de interação com 
Wnts. A via não-canônica também está envolvida em migração neuronal. Foram relatados pacientes com síndrome de Knobloch com falta apenas das isoformas média e longa que apresentam defeitos em migração neuronal e casos de epilepsia (PassosBueno, Suzuki et al., 2006). O envolvimento do COL18A1 na via não-canônica poderia representar um mecanismo integrado na migração neuronal.

Procuramos identificar elementos de regulação do COL18A1 e entender funcionalmente o papel do FRZC18 na via de sinalização Wnt, porém muitas questões ainda permanecem obscuras. Como por exemplo, os elementos de regulação em trans que se ligam às regiões funcionais identificadas neste trabalho ainda são desconhecidos. E também, a questão de como o FRZC18 funciona como antagonista da via de sinalização Wnt, assim como a possível interação física entre o FRZC18 e os Wnts, ainda permanecem para serem resolvidas. Estudos futuros podem elucidar pontos não resolvidos neste trablho ou novas questões surgidas durante o desenvolvimento do mesmo. 
Capítulo VI- Discussão

Capítulo VII 


\section{Referências Bibliográficas}

Abdollahi, A., P. Hahnfeldt, et al. Endostatin's antiangiogenic signaling network. Mol Cell, v.13, n.5, Mar 12, p.649-63. 2004.

Abe, N., Y. Muragaki, et al. Identification of a novel collagen chain represented by extensive interruptions in the triple-helical region. Biochem Biophys Res Commun, v.196, n.2, Oct 29, p.576-82. 1993.

Ackley, B. D., J. R. Crew, et al. The NC1/endostatin domain of Caenorhabditis elegans type XVIII collagen affects cell migration and axon guidance. J Cell Biol, v.152, n.6, Mar 19, p.1219-32. 2001.

Ahituv, N., E. M. Rubin, et al. Exploiting human--fish genome comparisons for deciphering gene regulation. Hum Mol Genet, v.13 Spec No 2, Oct 1, p.R261-6. 2004.

Antonellis, A., J. L. Huynh, et al. Identification of neural crest and glial enhancers at the mouse Sox 10 locus through transgenesis in zebrafish. PLoS Genet, v.4, n.9, Sep, p.e1000174. 2008.

Armelin-Correa, L. M., C. J. Lin, et al. Characterization of human collagen XVIII promoter 2: interaction of Sp1, Sp3 and YY1 with the regulatory region and a SNP that increases transcription in hepatocytes. Matrix Biol, v.24, n.8, Dec, p.550-9. 2005.

Baksh, D. e R. S. Tuan. Canonical and non-canonical Wnts differentially affect the development potential of primary isolate of human bone marrow mesenchymal stem cells. J Cell Physiol, v.212, n.3, Sep, p.817-26. 2007.

Bassett, E. A., G. F. Pontoriero, et al. Conditional deletion of activating protein 2alpha (AP-2alpha) in the developing retina demonstrates non-cell-autonomous roles for AP-2alpha in optic cup development. Mol Cell Biol, v.27, n.21, Nov, p.7497-510. 2007.

Bejerano, G., M. Pheasant, et al. Ultraconserved elements in the human genome. Science, v.304, n.5675, May 28, p.1321-5. 2004.

Bowers, R. R. e M. D. Lane. Wnt signaling and adipocyte lineage commitment. Cell Cycle, v.7, n.9, May 1, p.1191-6. 2008.

Carreira-Barbosa, F., M. L. Concha, et al. Prickle 1 regulates cell movements during gastrulation and neuronal migration in zebrafish. Development, v.130, n.17, Sep, p.4037-46. 2003.

Czeizel, A. E., P. Goblyos, et al. The second report of Knobloch syndrome. Am J Med Genet, v.42, n.6, Apr 1, p.777-9. 1992. 
Dann, C. E., J. C. Hsieh, et al. Insights into Wnt binding and signalling from the structures of two Frizzled cysteine-rich domains. Nature, v.412, n.6842, Jul 5, p.8690. 2001.

Doucas, H., G. Garcea, et al. Changes in the Wnt signalling pathway in gastrointestinal cancers and their prognostic significance. Eur J Cancer, v.41, n.3, Feb, p.365-79. 2005.

Elamaa, H., J. Peterson, et al. Cloning of three variants of type XVIII collagen and their expression patterns during Xenopus laevis development. Mech Dev, v.114, n.12, Jun, p.109-13. 2002.

Elamaa, H., A. Snellman, et al. Characterization of the human type XVIII collagen gene and proteolytic processing and tissue location of the variant containing a frizzled motif. Matrix Biol, v.22, n.5, Sep, p.427-42. 2003.

Elnitski, L., C. Riemer, et al. MultiPipMaker: comparative alignment server for multiple DNA sequences. Curr Protoc Bioinformatics, v.Chapter 10, Apr, p.Unit10 4. 2005.

Endo, Y., V. Wolf, et al. Wnt-3a-dependent cell motility involves RhoA activation and is specifically regulated by dishevelled-2. J Biol Chem, v.280, n.1, Jan 7, p.77786. 2005.

Errera, F. I., L. H. Canani, et al. COL18A1 is highly expressed during human adipocyte differentiation and the SNP c.1136C > T in its "frizzled" motif is associated with obesity in diabetes type 2 patients. An Acad Bras Cienc, v.80, n.1, Mar, p.16777. 2008.

Farmer, S. R. Regulation of PPARgamma activity during adipogenesis. Int J Obes (Lond), v.29 Suppl 1, Mar, p.S13-6. 2005.

Fisher, S., E. A. Grice, et al. Conservation of RET regulatory function from human to zebrafish without sequence similarity. Science, v.312, n.5771, Apr 14, p.276-9. 2006.

. Evaluating the biological relevance of putative enhancers using Tol2 transposon-mediated transgenesis in zebrafish. Nat Protoc, v.1, n.3, p.1297-305. 2006.

Fukai, N., L. Eklund, et al. Lack of collagen XVIII/endostatin results in eye abnormalities. EMBO J, v.21, n.7, Apr 2, p.1535-44. 2002.

Funayama, N., F. Fagotto, et al. Embryonic axis induction by the armadillo repeat domain of beta-catenin: evidence for intracellular signaling. J Cell Biol, v.128, n.5, Mar, p.959-68. 1995.

Gelse, K., E. Poschl, et al. Collagens--structure, function, and biosynthesis. Adv Drug Deliv Rev, v.55, n.12, Nov 28, p.1531-46. 2003. 
Grice, E. A., E. S. Rochelle, et al. Evaluation of the RET regulatory landscape reveals the biological relevance of a HSCR-implicated enhancer. Hum Mol Genet, v.14, n.24, Dec 15, p.3837-45. 2005.

Habas, R., Y. Kato, et al. Wnt/Frizzled activation of Rho regulates vertebrate gastrulation and requires a novel Formin homology protein Daam1. Cell, v.107, n.7, Dec 28, p.843-54. 2001.

Haftek, Z., G. Morvan-Dubois, et al. Sequence and embryonic expression of collagen XVIII NC1 domain (endostatin) in the zebrafish. Gene Expr Patterns, v.3, n.3, Jun, p.351-4. 2003.

Halfter, W., S. Dong, et al. Collagen XVIII is a basement membrane heparan sulfate proteoglycan. J Biol Chem, v.273, n.39, Sep 25, p.25404-12. 1998.

Hanai, J., J. Gloy, et al. Endostatin is a potential inhibitor of Wnt signaling. J Cell Biol, v.158, n.3, Aug 5, p.529-39. 2002.

Hayashi, Y., W. Wang, et al. Liver enriched transcription factors and differentiation of hepatocellular carcinoma. Mol Pathol, v.52, n.1, Feb, p.19-24. 1999.

He, T. C., A. B. Sparks, et al. Identification of c-MYC as a target of the APC pathway. Science, v.281, n.5382, Sep 4, p.1509-12. 1998.

Heisenberg, C. P. e M. Tada. Zebrafish gastrulation movements: bridging cell and developmental biology. Semin Cell Dev Biol, v.13, n.6, Dec, p.471-9. 2002.

Heisenberg, C. P., M. Tada, et al. Silberblick/Wnt11 mediates convergent extension movements during zebrafish gastrulation. Nature, v.405, n.6782, May 4, p.76-81. 2000 .

Holmen, S. L., A. Salic, et al. A novel set of Wnt-Frizzled fusion proteins identifies receptor components that activate beta -catenin-dependent signaling. J Biol Chem, v.277, n.38, Sep 20, p.34727-35. 2002.

Hong, S. Y., M. H. Lee, et al. Adeno-associated virus mediated endostatin gene therapy in combination with topoisomerase inhibitor effectively controls liver tumor in mouse model. World J Gastroenterol, v.10, n.8, Apr 15, p.1191-7. 2004.

$\mathrm{Hu}, \mathrm{B} ., \mathrm{L} . \mathrm{Kou}$, et al. Bifidobacterium longum as a delivery system of TRAIL and endostatin cooperates with chemotherapeutic drugs to inhibit hypoxic tumor growth. Cancer Gene Ther, Feb 20. 2009.

Hu, T. H., C. C. Huang, et al. Increased endostatin/collagen XVIII expression correlates with elevated VEGF level and poor prognosis in hepatocellular carcinoma. Mod Pathol, v.18, n.5, May, p.663-72. 2005.

Hurskainen, M., L. Eklund, et al. Abnormal maturation of the retinal vasculature in type XVIII collagen/endostatin deficient mice and changes in retinal glial cells due to lack of collagen types XV and XVIII. FASEB J, v.19, n.11, Sep, p.1564-6. 2005. 
Iizasa, T., H. Chang, et al. Overexpression of collagen XVIII is associated with poor outcome and elevated levels of circulating serum endostatin in non-small cell lung cancer. Clin Cancer Res, v.10, n.16, Aug 15, p.5361-6. 2004.

Katoh, M. Comparative integromics on non-canonical WNT or planar cell polarity signaling molecules: transcriptional mechanism of PTK7 in colorectal cancer and that of SEMA6A in undifferentiated ES cells. Int J Mol Med, v.20, n.3, Sep, p.405-9. 2007.

Kawano, Y. e R. Kypta. Secreted antagonists of the Wnt signalling pathway. J Cell Sci, v.116, n.Pt 13, Jul 1, p.2627-34. 2003.

Kawashima, H., N. Watanabe, et al. Collagen XVIII, a basement membrane heparan sulfate proteoglycan, interacts with L-selectin and monocyte chemoattractant protein1. J Biol Chem, v.278, n.15, Apr 11, p.13069-76. 2003.

Kelly, C., A. J. Chin, et al. Maternally controlled (beta)-catenin-mediated signaling is required for organizer formation in the zebrafish. Development, v.127, n.18, Sep, p.3899-911. 2000.

Kelly, G. M., D. F. Erezyilmaz, et al. Induction of a secondary embryonic axis in zebrafish occurs following the overexpression of beta-catenin. Mech Dev, v.53, n.2, Oct, p.261-73. 1995.

Kilian, B., H. Mansukoski, et al. The role of Ppt/Wnt5 in regulating cell shape and movement during zebrafish gastrulation. Mech Dev, v.120, n.4, Apr, p.467-76. 2003.

Kimmel, C. B., W. W. Ballard, et al. Stages of embryonic development of the zebrafish. Dev Dyn, v.203, n.3, Jul, p.253-310. 1995.

Kishida, S., H. Yamamoto, et al. Wnt-3a and Dvl induce neurite retraction by activating Rho-associated kinase. Mol Cell Biol, v.24, n.10, May, p.4487-501. 2004.

Kvist, A. P., A. Latvanlehto, et al. Complete exon-intron organization and chromosomal location of the gene for mouse type XIII collagen (col13a1) and comparison with its human homologue. Matrix Biol, v.18, n.3, Jun, p.261-74. 1999.

Lange, C., E. Mix, et al. Wnt signal pathways and neural stem cell differentiation. Neurodegener Dis, v.3, n.1-2, p.76-86. 2006.

Lele, Z., J. Bakkers, et al. Morpholino phenocopies of the swirl, snailhouse, somitabun, minifin, silberblick, and pipetail mutations. Genesis, v.30, n.3, Jul, p.1904. 2001.

Lien, C. L., J. Mcanally, et al. Cardiac-specific activity of an Nkx2-5 enhancer requires an evolutionarily conserved Smad binding site. Dev Biol, v.244, n.2, Apr 15, p.257-66. 2002. 
Logan, C. Y. e R. Nusse. The Wnt signaling pathway in development and disease. Annu Rev Cell Dev Biol, v.20, p.781-810. 2004.

Lourenco, G. J., C. Cardoso-Filho, et al. A high risk of occurrence of sporadic breast cancer in individuals with the 104NN polymorphism of the COL18A1 gene. Breast Cancer Res Treat, v.100, n.3, Dec, p.335-8. 2006.

Mann, B., M. Gelos, et al. Target genes of beta-catenin-T cell-factor/lymphoidenhancer-factor signaling in human colorectal carcinomas. Proc Natl Acad Sci U S A, v.96, n.4, Feb 16, p.1603-8. 1999.

Marlow, F., J. Topczewski, et al. Zebrafish Rho kinase 2 acts downstream of Wnt1 1 to mediate cell polarity and effective convergence and extension movements. Curr Biol, v.12, n.11, Jun 4, p.876-84. 2002.

Marneros, A. G. e B. R. Olsen. The role of collagen-derived proteolytic fragments in angiogenesis. Matrix Biol, v.20, n.5-6, Sep, p.337-45. 2001.

. Age-dependent iris abnormalities in collagen XVIII/endostatin deficient mice with similarities to human pigment dispersion syndrome. Invest Ophthalmol Vis Sci, v.44, n.6, Jun, p.2367-72. 2003.

. Physiological role of collagen XVIII and endostatin. FASEB J, v.19, n.7, May, p.716-28. 2005.

Mcgaughey, D. M., R. M. Vinton, et al. Metrics of sequence constraint overlook regulatory sequences in an exhaustive analysis at phox $2 \mathrm{~b}$. Genome Res, v.18, n.2, Feb, p.252-60. 2008.

Mi, K. e G. V. Johnson. Role of the intracellular domains of LRP5 and LRP6 in activating the Wnt canonical pathway. J Cell Biochem, v.95, n.2, May 15, p.328-38. 2005.

Montcouquiol, M., E. B. Crenshaw, 3rd, et al. Noncanonical Wnt signaling and neural polarity. Annu Rev Neurosci, v.29, p.363-86. 2006.

Muragaki, Y., S. Timmons, et al. Mouse Col18a1 is expressed in a tissue-specific manner as three alternative variants and is localized in basement membrane zones. Proc Natl Acad Sci U S A, v.92, n.19, Sep 12, p.8763-7. 1995.

Musso, O., M. Rehn, et al. Tumor progression is associated with a significant decrease in the expression of the endostatin precursor collagen XVIII in human hepatocellular carcinomas. Cancer Res, v.61, n.1, Jan 1, p.45-9. 2001.

Musso, O., N. Theret, et al. Tumor hepatocytes and basement membrane-Producing cells specifically express two different forms of the endostatin precursor, collagen XVIII, in human liver cancers. Hepatology, v.33, n.4, Apr, p.868-76. 2001.

Myllyharju, J. e K. I. Kivirikko. Collagens and collagen-related diseases. Ann Med, v.33, n.1, Feb, p.7-21. 2001. 
Nobrega, M. A., I. Ovcharenko, et al. Scanning human gene deserts for long-range enhancers. Science, v.302, n.5644, Oct 17, p.413. 2003.

Nobrega, M. A. e L. A. Pennacchio. Comparative genomic analysis as a tool for biological discovery. J Physiol, v.554, n.Pt 1, Jan 1, p.31-9. 2004.

O'reilly, M. S., T. Boehm, et al. Endostatin: an endogenous inhibitor of angiogenesis and tumor growth. Cell, v.88, n.2, Jan 24, p.277-85. 1997.

Oh, S. P., Y. Kamagata, et al. Isolation and sequencing of cDNAs for proteins with multiple domains of Gly-Xaa-Yaa repeats identify a distinct family of collagenous proteins. Proc Natl Acad Sci U S A, v.91, n.10, May 10, p.4229-33. 1994.

Oh, S. P., M. L. Warman, et al. Cloning of cDNA and genomic DNA encoding human type XVIII collagen and localization of the alpha 1(XVIII) collagen gene to mouse chromosome 10 and human chromosome 21. Genomics, v.19, n.3, Feb, p.4949. 1994.

Pashos, E. E., E. Kague, et al. Evaluation of cis-regulatory function in zebrafish. Brief Funct Genomic Proteomic, Sep 27. 2008.

Passos-Bueno, M. R., S. K. Marie, et al. Knobloch syndrome in a large Brazilian consanguineous family: confirmation of autosomal recessive inheritance. Am J Med Genet, v.52, n.2, Aug 15, p.170-3. 1994.

Passos-Bueno, M. R., O. T. Suzuki, et al. Mutations in collagen 18A1 and their relevance to the human phenotype. An Acad Bras Cienc, v.78, n.1, Mar, p.123-31. 2006.

Pederson, L., M. Ruan, et al. Regulation of bone formation by osteoclasts involves Wnt/BMP signaling and the chemokine sphingosine-1-phosphate. Proc Natl Acad Sci U S A, v.105, n.52, Dec 30, p.20764-9. 2008.

Pennacchio, L. A. Insights from human/mouse genome comparisons. Mamm Genome, v.14, n.7, Jul, p.429-36. 2003.

Ponti, G., L. Losi, et al. Wnt pathway, angiogenetic and hormonal markers in sporadic and familial adenomatous polyposis-associated juvenile nasopharyngeal angiofibromas (JNA). Appl Immunohistochem Mol Morphol, v.16, n.2, Mar, p.173-8. 2008.

Poulin, F., M. A. Nobrega, et al. In vivo characterization of a vertebrate ultraconserved enhancer. Genomics, v.85, n.6, Jun, p.774-81. 2005.

Prockop, D. J. e K. I. Kivirikko. Collagens: molecular biology, diseases, and potentials for therapy. Annu Rev Biochem, v.64, p.403-34. 1995.

Quelard, D., E. Lavergne, et al. A cryptic frizzled module in cell surface collagen 18 inhibits Wnt/beta-catenin signaling. PLoS ONE, v.3, n.4, p.e1878. 2008. 
Rehn, M., E. Hintikka, et al. Primary structure of the alpha 1 chain of mouse type XVIII collagen, partial structure of the corresponding gene, and comparison of the alpha 1 (XVIII) chain with its homologue, the alpha $1(\mathrm{XV})$ collagen chain. J Biol Chem, v.269, n.19, May 13, p.13929-35. 1994.

Characterization of the mouse gene for the alpha 1 chain of type XVIII collagen (Col18a1) reveals that the three variant $\mathrm{N}$-terminal polypeptide forms are transcribed from two widely separated promoters. Genomics, v.32, n.3, Mar 15, p.436-46. 1996.

Rehn, M. e T. Pihlajaniemi. Alpha 1(XVIII), a collagen chain with frequent interruptions in the collagenous sequence, a distinct tissue distribution, and homology with type XV collagen. Proc Natl Acad Sci U S A, v.91, n.10, May 10, p.4234-8. 1994.

Retsky, M. W., W. J. Hrushesky, et al. Hypothesis: primary antiangiogenic method proposed to treat early stage breast cancer. BMC Cancer, v.9, p.7. 2009.

Ross, S. E., N. Hemati, et al. Inhibition of adipogenesis by Wnt signaling. Science, v.289, n.5481, Aug 11, p.950-3. 2000.

Rosso, S. B., D. Sussman, et al. Wnt signaling through Dishevelled, Rac and JNK regulates dendritic development. Nat Neurosci, v.8, n.1, Jan, p.34-42. 2005.

Saarela, J., M. Rehn, et al. The short and long forms of type XVIII collagen show clear tissue specificities in their expression and location in basement membrane zones in humans. Am J Pathol, v.153, n.2, Aug, p.611-26. 1998.

Saarela, J., R. Ylikarppa, et al. Complete primary structure of two variant forms of human type XVIII collagen and tissue-specific differences in the expression of the corresponding transcripts. Matrix Biol, v.16, n.6, Jan, p.319-28. 1998.

Schneider, V. A. e M. Granato. The myotomal diwanka (lh3) glycosyltransferase and type XVIII collagen are critical for motor growth cone migration. Neuron, v.50, n.5, Jun 1, p.683-95. 2006.

Schwartz, S., L. Elnitski, et al. MultiPipMaker and supporting tools: Alignments and analysis of multiple genomic DNA sequences. Nucleic Acids Res, v.31, n.13, Jul 1, p.3518-24. 2003.

Sertie, A. L., M. Quimby, et al. A gene which causes severe ocular alterations and occipital encephalocele (Knobloch syndrome) is mapped to 21q22.3. Hum Mol Genet, v.5, n.6, Jun, p.843-7. 1996.

Sertie, A. L., V. Sossi, et al. Collagen XVIII, containing an endogenous inhibitor of angiogenesis and tumor growth, plays a critical role in the maintenance of retinal structure and in neural tube closure (Knobloch syndrome). Hum Mol Genet, v.9, n.13, Aug 12, p.2051-8. 2000. 
Shin, J. T., J. R. Priest, et al. Human-zebrafish non-coding conserved elements act in vivo to regulate transcription. Nucleic Acids Res, v.33, n.17, p.5437-45. 2005.

Suzuki, O. T., A. L. Sertie, et al. Molecular analysis of collagen XVIII reveals novel mutations, presence of a third isoform, and possible genetic heterogeneity in Knobloch syndrome. Am J Hum Genet, v.71, n.6, Dec, p.1320-9. 2002.

Taniguchi, K., L. R. Roberts, et al. Mutational spectrum of beta-catenin, AXIN1, and AXIN2 in hepatocellular carcinomas and hepatoblastomas. Oncogene, v.21, n.31, Jul 18, p.4863-71. 2002.

The ENCODE (ENCyclopedia Of DNA Elements) Project. Science, v.306, n.5696, Oct 22, p.636-40. 2004.

Thisse, C. e B. Thisse. High-resolution in situ hybridization to whole-mount zebrafish embryos. Nat Protoc, v.3, n.1, p.59-69. 2008.

Topczewski, J., D. S. Sepich, et al. The zebrafish glypican knypek controls cell polarity during gastrulation movements of convergent extension. Dev Cell, v.1, n.2, Aug, p.251-64. 2001.

Uchikawa, M., Y. Ishida, et al. Functional analysis of chicken Sox2 enhancers highlights an array of diverse regulatory elements that are conserved in mammals. Dev Cell, v.4, n.4, Apr, p.509-19. 2003.

Ulrich, F., M. L. Concha, et al. Slb/Wnt11 controls hypoblast cell migration and morphogenesis at the onset of zebrafish gastrulation. Development, v.130, n.22, Nov, p.5375-84. 2003.

Ulrich, F., M. Krieg, et al. Wnt11 functions in gastrulation by controlling cell cohesion through Rab5c and E-cadherin. Dev Cell, v.9, n.4, Oct, p.555-64. 2005.

Utriainen, A., R. Sormunen, et al. Structurally altered basement membranes and hydrocephalus in a type XVIII collagen deficient mouse line. Hum Mol Genet, v.13, n.18, Sep 15, p.2089-99. 2004.

Veeman, M. T., J. D. Axelrod, et al. A second canon. Functions and mechanisms of beta-catenin-independent Wnt signaling. Dev Cell, v.5, n.3, Sep, p.367-77. 2003.

Westerfield, M. The Zebrafish Book. A guide for the laboratory use of zebrafish (Danio rerio). Eugene: University of Oregon Press. 2000

Westfall, T. A., R. Brimeyer, et al. Wnt-5/pipetail functions in vertebrate axis formation as a negative regulator of Wnt/beta-catenin activity. J Cell Biol, v.162, n.5, Sep 1, p.889-98. 2003.

Woolfe, A., M. Goodson, et al. Highly conserved non-coding sequences are associated with vertebrate development. PLoS Biol, v.3, n.1, Jan, p.e7. 2005. 
$\mathrm{Wu}, \mathrm{J} ., \mathrm{D}$. Ding, et al. Sustained delivery of endostatin improves the efficacy of therapy in Lewis lung cancer model. J Control Release, Nov 19. 2008.

Zhang, X., J. P. Gaspard, et al. Regulation of vascular endothelial growth factor by the Wnt and K-ras pathways in colonic neoplasia. Cancer Res, v.61, n.16, Aug 15, p.6050-4. 2001.

Zorick, T. S., Z. Mustacchi, et al. High serum endostatin levels in Down syndrome: implications for improved treatment and prevention of solid tumours. Eur J Hum Genet, v.9, n.11, Nov, p.811-4. 2001. 